\section{Prolonged organ culture reduces the incidence of endothelial immune reactions}

P Maier, S Heinzelmann, D Böhringer and T Reinhard

\begin{abstract}
Purpose The number of antigen-presenting cells decreases during organ culture of corneoscleral discs. This might result in a decrease of immune reactions with increasing duration of organ culture. To investigate this hypothesis, we performed a retrospective analysis of all penetrating keratoplasties that were consecutively performed over the last 5 years.

Patients and methods All cases of penetrating keratoplasties $(n=1006)$ were divided into two groups, with the division made at the median of the storage time (21 days). These two groups were compared by a Cox proportional hazards survival model regarding the incidence of endothelial immune reactions, clear graft survival, and chronic endothelial cell loss following penetrating keratoplasty considering patient's age, donor's age, and risk situation as co-variates.

Results We observed statistically significantly fewer endothelial immune reactions $(20.1 \%$ (95\% confidence interval 15.5-24.5\%) after 2 years) in the group with a storage time of more than 21 days compared with the group with a storage time of $<21$ days $(26.5 \%$ (95\% confidence interval 21.6-31.2\%) after 2 years). However, the duration of organ culture did not have a statistically significant effect on clear graft survival or chronic endothelial cell loss. Conclusion Our results demonstrate that an increased duration of organ culture leads to a lower incidence of endothelial immune reactions following penetrating keratoplasty. However, we do not recommend increased storage times in general as overall graft survival did not improve. The reason for this apparent paradox may be that the endothelial cell count decreases during storage time.

Eye (2016) 30, 127-132; doi:10.1038/eye.2015.207; published online 23 October 2015

\section{Introduction}

Good long-term prognosis of penetrating corneal grafts has been attributed to the immunological privilege of the cornea and the anterior chamber. ${ }^{1}$ However, corneal graft rejection remains the most important reason for graft failure. ${ }^{2}$ Despite intense research, exact mechanisms leading to graft rejection are not yet fully understood. Besides the immunosuppressive climate in the anterior chamber, the mechanisms of antigen presentation inside and outside the cornea may have an important role in the development of endothelial immune reactions. In the cornea and on the ocular surface, dendritic cells (DCs), including Langerhans cells (LCs), and macrophages, are responsible for antigen presentation. ${ }^{3-7} \mathrm{By}$ immunostaining, different populations of APCs could be identified in the corneal epithelium as well as in the corneal stroma and not all of these cell types are HLA-DR positive. ${ }^{5}$ They serve as antigen-presenting cells (APC), recognising and destroying foreign tissue, cells, or molecules. These APCs have the capacity to determine the outcome of immunity or tolerance within the anterior ocular segment. ${ }^{8}$ It has been shown that the number of LCs and DCs slowly decreases during organ culture, ${ }^{9-13}$ although it seems that they do not vanish completely. Mayer et al ${ }^{13}$ found that human corneas harbour different populations of LCs and DCs in a distribution pattern similar to that in the skin. In their kinetic study of DCs in organ culture, they found a significant decrease of APCs within the first 3 weeks of organ culture. Epithelial APCs showed an almost linear slow decrease with a small proportion of HLA-DR-positive cells remaining there for up to 200 days, whereas stromal APCs showed the fastest decrease during the first 7 days of organ culture slowing down for the rest of storage. They concluded
\end{abstract}

Eye Centre, University Hospital Freibur, Germany

Correspondence: P Maier, Eye Centre, University Hospital Freibur, Killianstr. 5, 79106 Freiburg, Germany Tel: +49 761270 40060; Fax: +49 76127040630 E-mail: Philip.maier@ uniklinik-freiburg.de 
that these corneas may be less immunogenic after a certain period of storage. This was in agreement with clinical observations by Simon et al, ${ }^{14}$ where an increased graft survival was reported when corneas were cultured for $>7$ days.

As corneoscleral discs can now be stored for up to 36 days in organ culture, we wanted to investigate by a retrospective analysis whether the increased storage time (and the corresponding kinetics of APC reduction during organ culture) may further reduce the risk for immune reaction.

\section{Subjects and methods}

\section{Patients}

We included all cases of penetrating keratoplasty performed at our institution between 2004 and 2008 $(n=1006)$. We grouped these patients into those below $(n=506)$ and above $(n=501)$ the median of organ culture (rounded up to 21 days). Detailed data from the two study groups are given in Table 1 . To further analyse the effect of the kinetic decrease of corneal APCs during organ culture we divided the patients into three groups ( $<14$ days, $14-21$ days, $>21$ days of organ culture). The study was approved by the local ethics committee and all patients gave their informed consent that their clinical data may be used for scientific analyses.

\section{Penetrating keratoplasty, postoperative therapy, follow-up}

All keratoplasties were performed with mechanical trephines. To fix the grafts, we used double running crossstitch sutures ${ }^{15}$ with Nylon 10.0. Gentamycin ointment was administered following surgery at least until the graft was covered by a complete epithelial layer. Then, prednisolone-21-acetate $1 \%$ eye drops were given five times daily and tapered during the first 5 postoperative months. Systemic corticosteroids were administered for only 3 weeks postoperatively. Oral acetazolamide was prescribed at a daily dose of $2 \times 250 \mathrm{mg}$ for 5 days postoperatively. Follow-up examinations of the graft were scheduled 6 weeks, and 4,12, and 18 months postoperatively, and thereafter once a year.

High-risk cases were defined by an increased risk for immune reactions, as described elsewhere. ${ }^{16}$

\section{Grafts}

Grafts were preserved in organ culture according to the guidelines of the European Eye Bank Association. After in situ corneoscleral excision the corneoscleral discs were stored in DMEM-based organ culture media containing $2 \%$ of newborn calf serum at $34^{\circ} \mathrm{C}$. Every 7 days the medium was renewed. Before transplantation the grafts were stored for 1-4 days in the same culture medium that additionally contained 5\% of high molecular dextrane to deswell the corneas for surgery. After 7 days and before transplantation the grafts were put into hypotonic buffered saline solution for endothelial cell analysis using a phase contrast microscope. More information on donor and graft properties like post-mortem times and donor age are given in Table 1.

\section{Immune reactions}

Endothelial immune reactions were diagnosed clinically via endothelial immune precipitates with accompanying stromal oedema at the slit lamp.

\section{Statistics}

For statistical analysis, all computations were performed using the R-Software system. ${ }^{17}$ We performed a Cox

Table 1 Clinical data on both study groups

\begin{tabular}{|c|c|c|c|}
\hline & $\begin{array}{c}\text { Group } 1(\mathrm{n}=506) \\
\leq 21 \text { days }\end{array}$ & $\begin{array}{c}\text { Group } 2(\mathrm{n}=501) \\
>21 \text { days }\end{array}$ & P-value \\
\hline Storage time $($ mean $\pm \mathrm{SD})$ & $16.8 \pm 3$ days & $24.4 \pm 2,7$ days & $<0.001$ \\
\hline Post-mortem time $($ mean $\pm \mathrm{SD})$ & $27 \pm 15$ days & $28 \pm 17$ days & 0.98 \\
\hline Donor age $($ mean $\pm \mathrm{SD})$ & $64 \pm 17$ years & $63 \pm 18$ years & 0.47 \\
\hline Patient age $($ mean $\pm S D)$ & $59 \pm 19$ years & $58 \pm 19$ years & 0.50 \\
\hline Follow-up (median, lower, and upper quartile) & $540(264,848)$ days & $509(264,809)$ days & 0.34 \\
\hline Percentage of normal risk patients & $31 \%(n=159)$ & $32 \%(n=158)$ & 0.97 \\
\hline Percentage of matched grafts ( $\leq 2$ mismatches $)^{a}$ & $34 \%$ & $33 \%$ & 0.83 \\
\hline Immune reaction after 2 years ${ }^{\mathrm{b}}$ (percentage, $95 \%$ confidence interval) & $26.5(21.6-31.2) \%$ & $20.1(15.5-24.5) \%$ & NA \\
\hline Immune reaction after 3 years $^{\mathrm{b}}$ (percentage \pm 95 confidence interval) & $32.6(25.9-38.6) \%$ & $25.9(18.9-32.3) \%$ & NA \\
\hline
\end{tabular}

There was a statistical significant difference regarding the occurrence of endothelial immune reactions between the two groups. All other parameters were

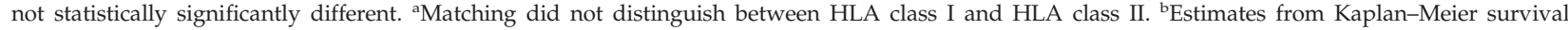
analysis. 
proportional hazards model including three co-variates: storage time, patient's age at time of surgery, and the risk status of the patient. Furthermore, we performed KaplanMeier survival estimations of event-free survival times. $P$-values $<0.05$ were regarded as statistically significant.

\section{Results}

We observed more immune reactions in the group of patients who received grafts with a storage time of $\leq 21$ days (Figure 1 ) and this was confirmed in the Cox regression model $(P=0.03)$. After 2 years estimates from Kaplan-Meier survival analysis were 26.5\% (95\% confidence interval $21.6-31.2 \%$ ) of grafts with an endothelial immune reaction in Group 1 (storage time $<21$ days), compared with $20.1 \%$ (95\% confidence interval 15.5-24.5\%) in Group 2 (storage time > 21 days). After 3 years the respective estimates from Kaplan-Meier survival analysis were $32.6 \%$ (95\% confidence interval 25.9-38.6\%) for Group 1 and 25.9\% (95\% confidence interval 18.9-32.3\%) for Group 2 (see Table 1). However, the storage time did not have a significant effect on the overall clear graft survival or on the relative annual endothelial cell loss following penetrating keratoplasty (figure 2, Cox regression analysis for clear graft survival $P=0.78$, Kruskal-Wallis test for the relative annual endothelial cell loss $P=0.89$ ).

To find out whether the specific kinetics of the APC decrease during organ culture may have an influence on the rate of immune reactions we divided the patients into three groups: patients who received grafts that have been stored for $<14$ days or 14-21 days or $>21$ days. Comparing the three groups Kaplan-Meier survival analysis revealed that the group with grafts stored $<14$ days had the highest risk for an immune reaction compared with the other two groups (Figure 3).

Various other parameters (eg, age of the recipients at time of surgery, percentage of high-risk keratoplasties, percentage of HLA-matched grafts) that might influence the occurrence of immune reactions were comparable in both study groups without any statistically significant difference (Table 1).

\section{Discussion}

Cold storage or short-term storage that is used, for example, in US eye banks to preserve corneal grafts allows a maximum storage time of 14 days at $4{ }^{\circ} \mathrm{C}$. Using organ culture as it is practiced by most European eye banks, corneal grafts are kept in cell culture media at $34-37^{\circ} \mathrm{C}$ for up to 36 days. However, it has been shown that increasing storage times in both preservation systems lead to reduced endothelial cell counts. ${ }^{18}$ Therefore, in most eye banks, a shorter storage time is preferred.

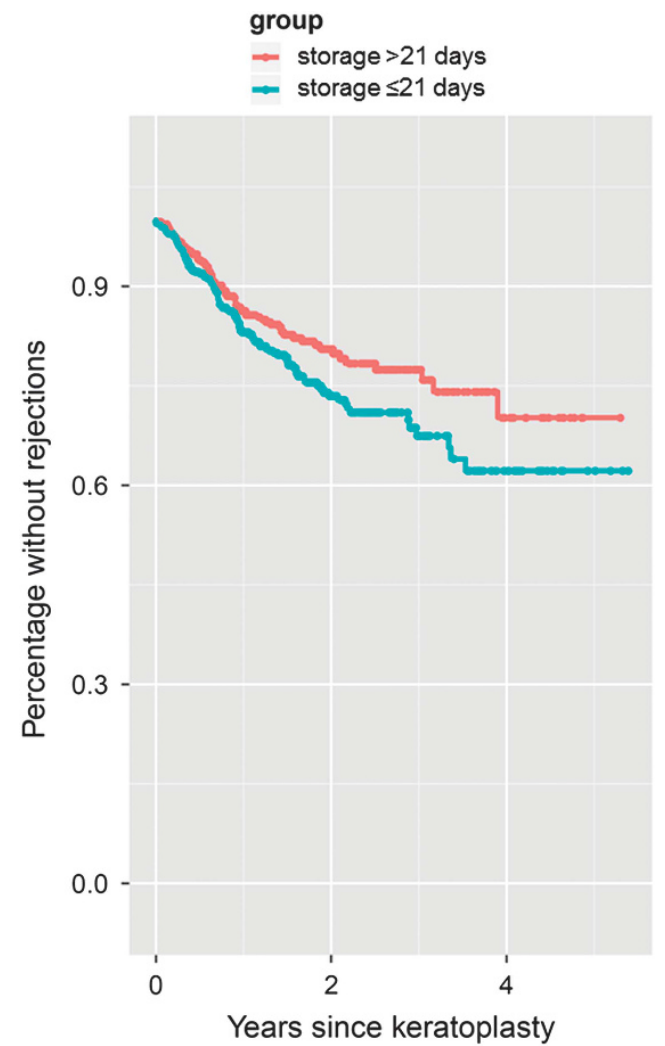

Figure 1 Kaplan-Meier survival analysis regarding the occurrence of immune reactions. Patients who received a graft that was $>21$ days in organ culture showed statistically significantly fewer immune reactions compared to patients with grafts of $\leq 21$ days of organ culture (Cox regression analysis $P=0.03$ ). After 2 years estimates from Kaplan-Meier survival analysis were 26.5 (95\% confidence interval $21.6-31.2) \%$ of grafts with an endothelial immune reaction in Group 1 (storage time $<21$ days), compared with 20.1 (95\% confidence interval 15.5-24.5)\% in Group 2 (storage time $>21$ days). After 3 years the respective estimates from Kaplan-Meier survival analysis were 32.6 (95\% confidence interval $25.9-38.6) \%$ for Group 1 and 25.9 (95\% confidence interval 18.9-32.3)\% for Group 2 (see Table 1).

For organ culture, the minimum storage time is typically 10-14 days due to quarantine requirements. On the other hand, it has been found that during organ culture the number of APCs in the corneoscleral discs slowly decreases. ${ }^{9-13}$ Pels et $a l^{9}$ and Ardjomand et al ${ }^{10,11}$ looked only for HLA-DR positivity in their analyses and found a complete vanishing (Pels et $a l^{9}$ ) or a significant reduction (Ardjomand et $a l^{10,11}$ ) of HLA-DR-positive DCs within 7 days of organ culture, which was independent of the storage media. More recently Al-Fakih et al ${ }^{12}$ also looked for HLA-DR-positive cells in corneoscleral discs and found that the decrease of these cells was faster when corneas were stored in organ culture compared with hypothermic storage. They also reported on an almost complete loss of HLA-DR-positive cells in the corneal epithelium within 14 days of organ culture, which took a 


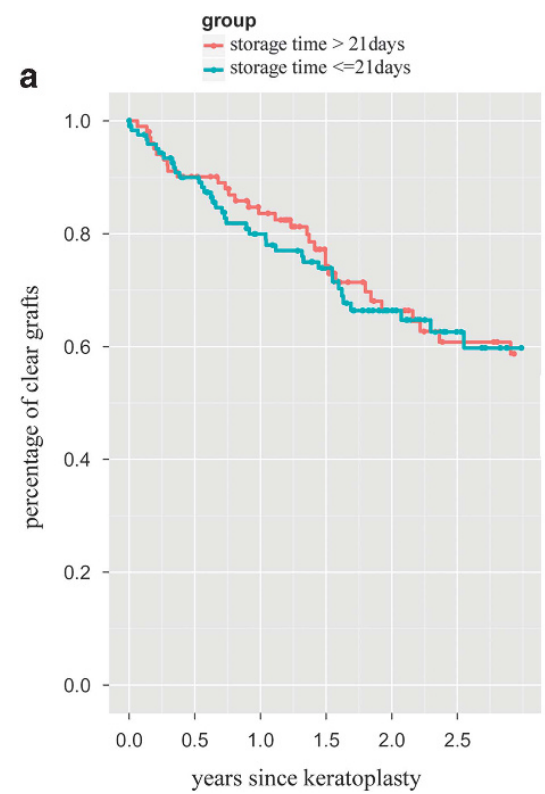

b

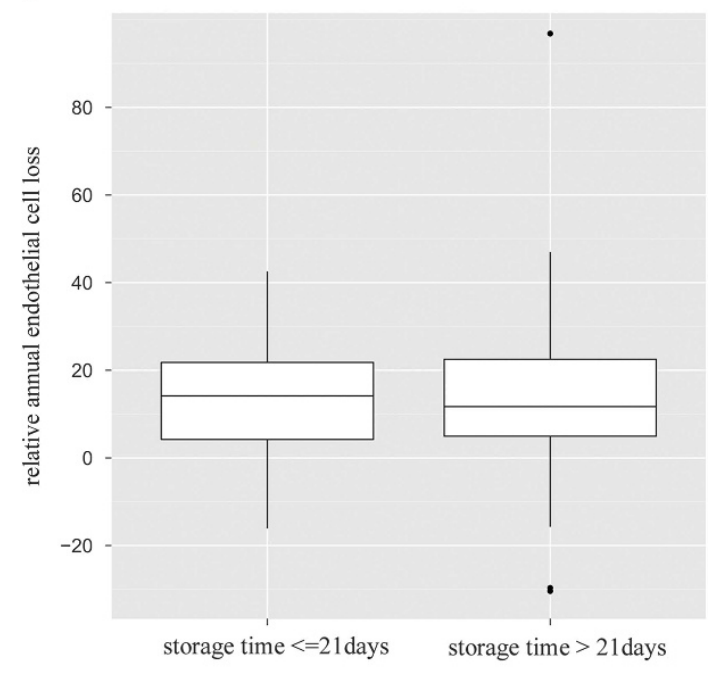

Figure 2 (a) Kaplan-Meier survival analysis regarding clear graft survival. There is no difference between the group of patients who received a graft that was $>21$ days in organ culture and patients with grafts of $\leq 21$ days of organ culture. Cox regression analysis $P=0.78$. (b) Box-plot diagram of the relative annual endothelial cell loss between the two study groups showing no difference between grafts with longer ( $>21$ days) or shorter ( $\leq 21$ days) storage time. Kruskal-Wallis test. $P=0.89$.

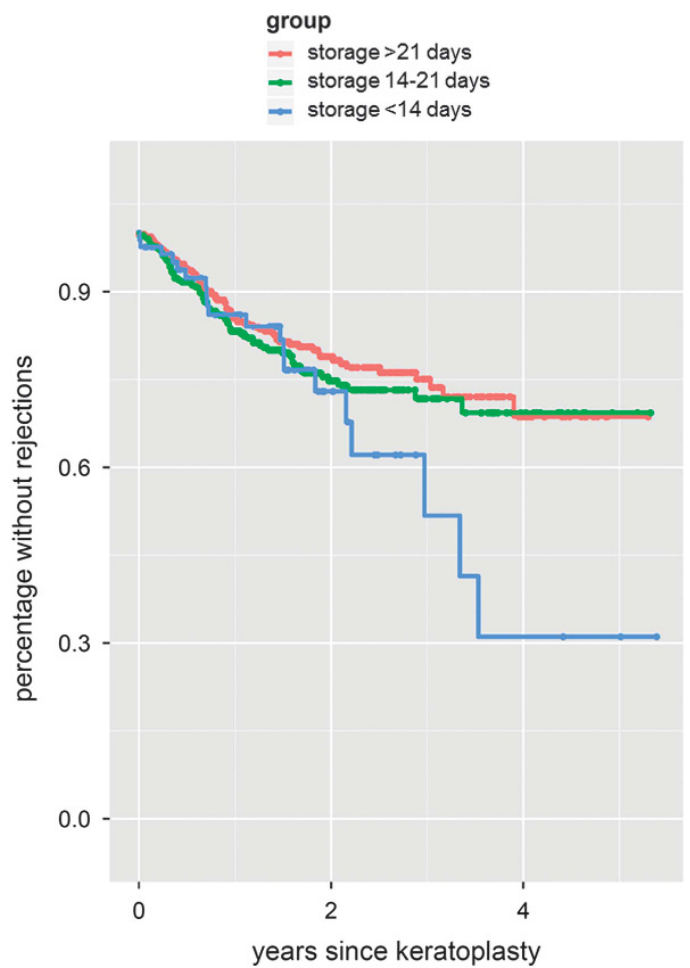

Figure 3 Kaplan-Meier analysis regarding the occurrence of immune reactions with respect to the kinetics of APC decrease during organ culture. Patients who received a graft that was stored for $<14$ days in organ culture showed more immune reactions compared with patients with grafts of $>14$ or $>21$ days of organ culture. little longer in the corneal stroma. As HLA-DR obviously is not the only marker to detect $\mathrm{APCs}^{5}$ it cannot be concluded from these reports that all corneal APCs finally disappear. Therefore, Mayer $e t a l^{13}$ performed a moredifferentiated analysis where they found a significant number of HLA-DR-positive LCs (Langerin+/CD1a+/ HLA-DR+) in the limbal and peripheral regions of corneal epithelium and the anterior stroma but also a significant number of HLA-DR-negative DCs (DCSIGN+/CD45+) mainly peripherally and in the anterior stroma but not in the epithelium. They also found mature DCLAMP+ and HLA-DR+ cells mainly in the stroma. Furthermore, they found two other populations of APCs (CD45+/CD11c - / HLA-DR-/DCLAMP-/Langerin-/CD1a-/DCSIGN- in the epithelium and CD45+/CD11c- /HLA-DR+/ Langerin-/CD1a-/DCSIGN- in the stroma). During longterm organ culture they found a slow almost linear loss of Langerin,$+ \mathrm{CD} 1 \mathrm{a}+$ and DCLAMP+ cells in the corneal epithelium, whereas a significant portion of HLA-DR+ cells survived for up to 200 days of organ culture. In the stroma the highest decrease of DCSIGN+, DCLAMP+ and HLA-DR+ cells was observed within the first 7 days of organ culture, whereas the respective cell number decreased much slower in the following long-term storage.

In an animal model of corneal transplantation, it has been shown that selective depletion of APCs in murine corneas leads to increased graft survival. ${ }^{19-21}$ Furthermore, it has been found that the reduction of 
APCs during corneal storage may lead to a decreased risk of immune reactions and even to increased clear graft survival rates. ${ }^{14}$ In our retrospective analysis, we found that even longer storage times of $>21$ days of organ culture led to a statistically significant decreased risk of immune reactions. From the in vitro results of the kinetics regarding the stromal APC decrease with the fastest depletion of stromal APCs during the first 7 days of organ culture $^{13}$ and our observation that the highest rate of rejections was in the group of grafts with storage times below 14 days compared with longer storage periods (see Figure 3) it might be concluded that stromal and not only epithelial DCs have an important role in corneal allograft rejection.

Simon et $a l^{14}$ reported in their retrospective clinical study that a storage time of 7 days or more has been observed to be associated with a beneficial effect on allograft survival. They also found that the rate of allograft rejection was significantly lower for patients receiving donor corneas after prolonged storage ( $>7$ days). However, this was only statistically significant for high-risk (not for normal risk) patients. In their study, the number of graft rejections in the low-risk group, which comprised only 135 patients was probably underpowered, resulting in a non-significant effect.

However, it is not clear whether the reduction of APCs is the correct explanation for the graft-protecting effect of prolonged organ culture. Nevertheless, direct and indirect allosensitization seem to have an important role in corneal allograft rejection. ${ }^{4-6,22}$ Even small numbers of these cells found in the mouse cornea were able to induce corneal allosensitization. ${ }^{23}$ Furthermore, it has been shown that donor-derived corneal APCs can be found in the draining lymph node and the spleen of the recipient within only a few hours following corneal transplantation. ${ }^{6}$ Rejection episodes following renal transplantations, for example, were reduced after inactivation of donor-derived hematopoietic cells, leading to an increased allograft survival time. ${ }^{24}$ Reduction of APCs in the cornea may be easily achieved by prolonged organ culture as compared with renal grafts, where the kidneys need to be perfused with anti-CD45 antibodies.

Besides the number of APCs, the endothelial cell density also decreases over time during organ culture. This difference in endothelial cell density can be found in the recipients for at least 1 year following penetrating keratoplasty. ${ }^{25}$ Therefore, a shorter storage time might be correlated with longer clear graft survival if the patient does not experience an endothelial immune reaction. Therefore, in normal risk situations, shorter storage times should be preferred.

Besides the decrease of APCs and the decrease of endothelial cells in the graft during organ culture, various other factors, like the clinical risk profile of the patients,
HLA-matching, postoperative immunosuppression, or the compliance of the patients, need to be considered regarding the individual rejection risk and clear graft survival. Therefore, the optimum storage time with respect to the patient's overall graft prognosis needs to be defined by the eye bank and the surgeon.

\section{Summary}

What was known before

- Endothelial immune reactions are the major cause of graft failure following penetrating keratoplasty.

- During organ culture the number of APCs in corneoscleral discs is decreasing over time.

What this study adds

- As the number of APCs in corneal grafts is decreasing during organ culture storage the risk of endothelial immune reactions can be lowered by increasing the storage time.

- This might be especially important in high-risk situations when systemic immunosuppression in these patients may be avoided.

\section{Conflict of interest}

The authors declare no conflict of interest.

\section{Acknowledgements}

All authors met the following criteria regarding their contribution to this work: (1) substantial contributions to conception and design, or acquisition of data, or analysis and interpretation of data; (2) drafting the article or revising it critically for important intellectual content; (3) final approval of the version to be published. The corresponding author Philip Maier had full access to all the data in the study and takes responsibility for the integrity of the data and the accuracy of the data analysis.

\section{References}

1 Niederkorn JY. Immunology and immunomodulation of corneal transplantation. Int Rev Immunol 2002; 21(2-3): 173-196.

2 Claesson M, Armitage WJ, Fagerholm P, Stenevi U. Visual outcome in corneal grafts: a preliminary analysis of the Swedish Corneal Transplant Register. Br J Ophthalmol 2002; 86(2): 174-180.

3 Hamrah P, Huq SO, Liu Y, Zhang Q, Dana MR. Corneal immunity is mediated by heterogeneous population of antigen-presenting cells. J Leukoc Biol 2003; 74(2): 172-178.

4 Hamrah P, Liu Y, Zhang Q, Dana MR. The corneal stroma is endowed with a significant number of resident dendritic cells. Invest Ophthalmol Vis Sci 2003; 44(2): 581-589. 
5 Hamrah P, Zhang Q, Liu Y, Dana MR. Novel characterization of MHC class II-negative population of resident corneal Langerhans cell-type dendritic cells. Invest Ophthalmol Vis Sci 2002; 43(3): 639-646.

6 Liu Y, Hamrah P, Zhang Q, Taylor AW, Dana MR. Draining lymph nodes of corneal transplant hosts exhibit evidence for donor major histocompatibility complex (MHC) class II-positive dendritic cells derived from MHC class IInegative grafts. J Exp Med 2002; 195(2): 259-268.

7 Niederkorn JY. Immune privilege in the anterior chamber of the eye. Crit Rev Immunol 2002; 22: 1.

8 Novak N, Siepmann K, Zierhut M, Bieber T. The good, the bad and the ugly-APCs of the eye. Trends Immunol 2003; 24(11): 570-574.

9 Pels E, van der Gaag R. HLA-A,B,C, and HLA-DR antigens and dendritic cells in fresh and organ culture preserved corneas. Cornea 1984; 3(4): 231-239.

10 Ardjomand N, Berghold A, Reich ME. Loss of corneal Langerhans cells during storage in organ culture medium, Optisol and McCarey-Kaufman medium. Eye (Lond) 1998; 12 (Pt 1): 134-138.

11 Ardjomand N, Komericki P, Radner H, Aigner R, Reich ME. [Corneal Langerhans cells. Behavior during storage in organ culture]. Ophthalmologe 1997; 94(10): 703-706.

12 Al-Fakih A, Faltus V, Jirsova K. A decrease in the density of HLA-DR-positive cells occurs faster in corneas stored in organ culture than under hypothermic conditions. Ophthalmic Res 2012; 47(1): 39-46.

13 Mayer WJ, Irschick UM, Moser P, Wurm M, Huemer HP, Romani $\mathrm{N}$ et al. Characterization of antigen-presenting cells in fresh and cultured human corneas using novel dendritic cell markers. Invest Ophthalmol Vis Sci 2007; 48(10): 4459-4467.

14 Simon M, Fellner P, El-Shabrawi Y, Ardjomand N. Influence of donor storage time on corneal allograft survival. Ophthalmology 2004; 111(8): 1534-1538.

15 Hoffmann F. [Suture technique for perforating keratoplasty (author's transl)]. Klin Monatsbl Augenheilkd 1976; 169(5): 584-590.
16 Reinhard T, Mayweg S, Sokolovska Y, Seitz B, Mittelviefhaus H, Engelmann $\mathrm{K}$ et al. Systemic mycophenolate mofetil avoids immune reactions in penetrating high-risk keratoplasty: preliminary results of an ongoing prospectively randomized multicentre study. Transpl Int 2005; 18(6): 703-708.

17 Ihaka R, Gentleman R. R: A Language for Data Analysis and Graphics. J Comput Graph Stat 1996; 5(3): 299-314.

18 Pels E, Rijneveld WJ. Organ culture preservation for corneal tissue. Technical and quality aspects. Dev Ophthalmol 2009; 43: 31-46.

19 He YG, Niederkorn JY. Depletion of donor-derived Langerhans cells promotes corneal allograft survival. Cornea 1996; 15(1): 82-89.

20 Kamiya K, Hori J, Kagaya F, Usui T, Amano S, Oshika T et al. Preservation of donor cornea prevents corneal allograft rejection by inhibiting induction of alloimmunity. Exp Eye Res 2000; 70(6): 737-743.

21 Kamiya K, Hori J, Obata H, Yamagami S, Tsuru T. Incidence of allograft rejection after corneal transplantation using optisol-preserved corneas in mice. Transplant Proc 1999; 31(6): 2673-2674.

22 Bohringer D, Daub F, Schwartzkopff J, Maier P, Birnbaum F, Sundmacher $\mathrm{R}$ et al. Operational post-keratopasty graft tolerance due to differential HLAMatchmaker matching. Mol Vis 2010; 16: 2362-2367.

23 Nichols J, Steger-May K, Edrington TB, Zadnik K. The relation between disease asymmetry and severity in keratoconus. Br J Ophthalmol 2004; 88(6): 788-791.

24 Goldberg LC. Pretreatment of kidney allografts with monoclonal antibodies to CD45: results of a multicentre study. CD45 Study Group. Transpl Int 1994; 7(Suppl 1): S252-S254.

25 Thuret G, Chiquet C, Bernal F, Acquart S, Romanet J-P, Mouillon $\mathrm{M}$ et al. Prospective, randomized clinical and endothelial evaluation of 2 storage times for cornea donor tissue in organ culture at 31 degrees C. Arch Ophthalmol 2003; 121(4): 442-450. 